\title{
Morpho-Diversification Study of Indigenous Gene-Pool of Pisum sativum L. at Madhya Pradesh, India
}

\author{
Lokesh Gour*, R.K. Dubey, S.K. Singh and Sharad Tiwari
}

Department of Plant Breeding and Genetics, Jawaharlal Nehru Krishi Vishwa Vidyalaya, Jabalpur (M.P.) 482004, India

*Corresponding author

\begin{tabular}{|c|}
\hline Keywords \\
\hline $\begin{array}{l}\text { Pisum sativum, Gene- } \\
\text { pool, Variation, } \\
\text { Unique germplasm, } \\
\text { Candidate variety }\end{array}$ \\
\hline Article Info \\
\hline $\begin{array}{l}\text { Accepted: } \\
20 \text { February } 2018 \\
\text { Available Online: } \\
10 \text { March } 2018\end{array}$ \\
\hline
\end{tabular}

\section{A B S T R A C T}

The goal of the study is to evaluate the major characteristics of pea (Pisum sativum L.) accessions to analyse morphological genetic diversity and identify the candidate genotypes from indigenous gene-pool of Pisum sativum L. The present investigation will be conducted at seed breeding farm, college of agriculture, Jabalpur in randomized complete block design with three replications on 101 pea genotypes for morpho-diversification study of 13 attributing characters. Rabbit eared stipule, compound tendril type, leaflets presence, foliage colour, pod shape of distal part, seed cotyledon colour, seed shape and seed surface has high variation among all germplasm lines. Leaf axil and flower standard petal colour were found purple in 7 lines namely B-22, DDR-54, JP-885 (Purple), FP 14-67, Kalamatar, FP 14-67, FP 14-88 so may be concern that genes governing leaf axil colour also express for flower standard petal colour. Above study revealed the distinct characteristics of germplasms and indicated that morphological variations exist in collected germplasms due to variation in genetic makeup and could be better utilized by breeders in the selection based on their specific requirement for breeding programme along with that this is highly useful study for varietal identification and conservation. The unique germplasms were found; B-22, FP 14-67, FP 14-88 which has stem anthocyanin coloration whereas DDR-23 and FP7-562 lines found normal pod curvature. Further, the unique features can be selected for developing varieties with unique identification may be particularly referred to as indicators to determine an unstable expression of the phenotype of candidate variety.

\section{Introduction}

Pulses occupy an important position in the agrarian economy of our country. Among the pulses crops grown in India, field pea (Pisum sativum L.) belonging to family Leguminoceae is cultivated for green pod seeds as vegetable and for dry seeds (Basaiwala et al., 2013).
Grains of pea are rich source of proteins, carbohydrates and other vitamins, which are good for human and livestock consumption. Dry pea grains are naturally dried ones that are consumed in soups, stews and various other cuisines (Parihar et al., 2014). Pea is an excellent source of protein $(27.8 \%)$, carbohydrates $(42.65 \%)$, vitamins, minerals, dietary fibres and antioxidant compounds 
(Gopalakrishnan, 2007). The major field pea producing states are UP, MP, Bihar, Assam and Orissa which together account for about $95 \%$ of the total area and production of pea in India. Baranger et al., (2004) proposed that frequent use of few parents in breeding programs has led to a low genetic diversity among pea varieties, but Nisar et al., (2011) found a considerable level of variance available to the breeders and proposed that genotypes from different origins can be utilized for genetic improvement without losing genetic diversity in pea. The success of genetic improvement is attributed to the magnitude and nature of variability present for a specific trait and the effectiveness of a breeding program largely depends on the existence of genetic variability, so a diverse genetic background provides desirable allelic variation among parental lines to produce new and valuable combinations (Tar'an et al., 2005). Qualitative characters are considered as morphological markers in the identification of landraces of rice, because they are less influenced by environmental changes (Raut, 2003) and the most common approach utilized to estimate relationships between genotypes (Bajracharya et al., 2006).

Since several morphological characters (descriptors,) remain the only legitimate marker types accepted by the International Union for the Protection of New Varieties of Plants (Tar'an et al., 2005 and UPOV, 1990), the analysis of morphological diversity among pea varieties and local populations in this study is realized based on characterization and evaluation of qualitative characters.

\section{Materials and Methods}

Exploration and collection of pea germplasms

In this research focus were on field and vegetable pea lines both. Experimental material consists of one hundred one genotype of pea was received from Field Pea Improvement Project, Department of Plant Breeding and Genetics, COA, Jabalpur and AICRP on MULLaRP, IIPR Kanpur.

\section{Evaluation of pea germplasms}

One hundred one germplasm lines and released varieties of pea were planted during Rabi season at the Seed Breeding Farm, Department of Plant Breeding and Genetics, COA, Jabalpur (M.P.), India. The material was grown in a complete randomized block design with three replications. Each entry was sown in three rows of $4 \mathrm{~m}$ length at spacing of $30 \mathrm{~cm}$ between rows and $10 \mathrm{~cm}$ between plants. The observation of various characteristics was recorded at different stages of growth with appropriate procedures as per the DUS test guidelines of PPV \& FR Authority, GOI, New Delhi are given here.

\section{Characterization and grouping of germplasm for qualitative traits}

Thirteen morphological traits on five randomly chosen plants of each line per replication were observed and characterized as per their note number given according to the National Test Guidelines for DUS test of Pea (Table 1 and 2), which was developed by PPV and FR Authority, GOI, New Delhi.

The candidate lines for DUS testing were divided into groups to facilitate the assessment of distinctness. On the basis of pigment distribution in 5 different plant parts, the entire germplasm was classified into some of groups; this will help in identification and further selection of lines for pigmentation study because it is rarely known that pigment has linkage with some of important yield and quality related traits. Unique accessions are identified as per their pigmentation found in specific parts. 


\section{Results and Discussion}

Morphological characterization is an important and basic methodology to establish distinctiveness among the genotypes. Thirteen traits were observed in one hundred one germplasm to establish distinctiveness among germplasm and these morphological traits play key role in varietal identification (Table 2).

\section{Characterization of the Qualitative Characters}

Foliage waxy bloom and normal stipule type were found in all 101 germplasms which didn't find any variation in both the traits (Table 2). Stem anthocyanin coloration were present in 3 lines namely B-22, FP 14-67, FP 14-88 but other all $98(97.03 \%)$ lines absent. Normal pod curvature found in 2 lines namely DDR-23 and FP 7-562 but 40 lines has weak and 59 lines has absent curvature which showed high variation. Similarly, high variation also found in foliage colour where 24 light green, 29 green and 48 dark green lines resulted. Variation found higher for other traits also as leaflets were absent in 22 and present in 79 germplasm lines. Stipule rabbit eared found present in 37 lines rest of lines has absentia (Fig. 1 and 2).

Tendril type were found Compound in 23 lines rest of all have normal tendril. Similarly, 19 lines were cylindrical, 12 lines were dimpled and rest of 70 lines has spherical seed shape. Seed surface of 21 lines were wrinkled and rest of 80 lines has smooth surface. Whereas, seed cotyledon colour were got creamy in 46, green in 8 and yellow in 47 lines. Pod shape of distal part pointed were in lines namely PP-96, FP 14-76, Kalamatar, FP 14-44, FP 14-51, FP 14-17, FP 14-23 but rest of 94 lines has blunt shape. Leaf axil color (Fig. 3 and 4) and flower standard petal colour were found purple in 7 lines namely B-22, DDR-54, JP-885(Purple), FP 14-67,
Kalamatar, FP 14-67, FP 14-88, those 7 germplasms having colour on leaf axil and flower standard petal both showing some different result in such condition.

\section{Grouping of Germplasms in respect of Pigmentation in Plant Parts}

Variations in degree of pigmentation suggest few or more gene controlling above traits. Some genotypes clearly differ from one another, for their pigmentation. On the basis of pigment distribution in 5 plant parts, the entire germplasm was classified into 15 groups, with group I to VI having pigmentation in only 1 germplasm whereas group XV with pigmentation in 24 germplasms (Table 3 ).

Total 6 small groups have had only 1 germplasm each (B-22, DDR 54, JP 885 (purple), FP 14-67, Kalamatar, RP 3) including a different group of traits. Group VII $\&$ VIII has only 2 germplasm each whereas group IX has included 3 germplasms. Largest group XV included 24 germplasms having traits associated with colour as absent stem anthocyanin colour, dark green foliage colour, white-flower standard petal colour, green-leaf axil colour and creamy-seed cotyledon colour.

\section{Unique identified pea germplasms}

The studied qualitative agro-morphological characters showed wide degree of variations among the lines. Out of 101 germplasm, stem anthocynin coloration were present in 3 germplasm namely B-22, FP 14-67, FP 14-88 and normal pod curvature found in 2 germplasm only DDR-23 and FP 7-562 so those traits can be considered as indicator for varietal identification. Leaf axil color (Fig. 3 and 4) and flower standard petal colour were found purple in 7 lines namely B-22, DDR-54, JP-885(Purple), FP 14-67, Kalamatar, FP 1467, FP 14-88. 
Fig.1 Stipule rabbit eared absent.

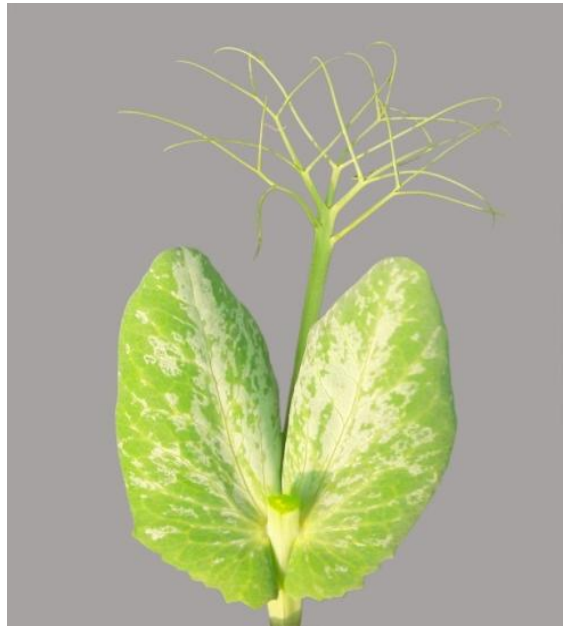

Fig.3 Stipule axil colour purple

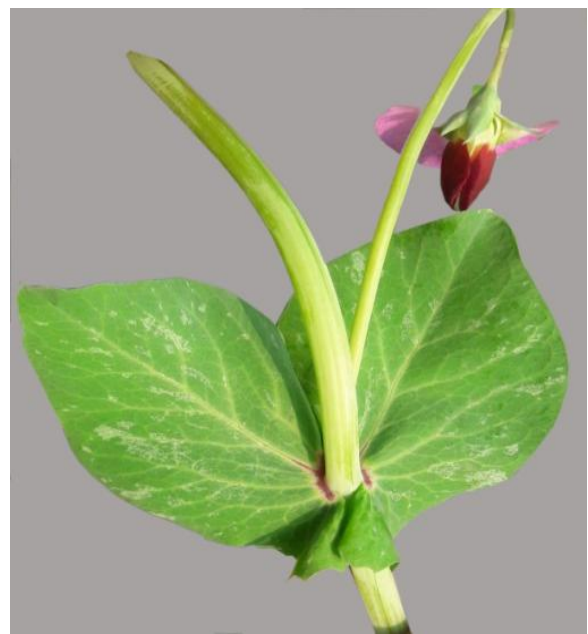

Fig.2 Stipule rabbit eared present

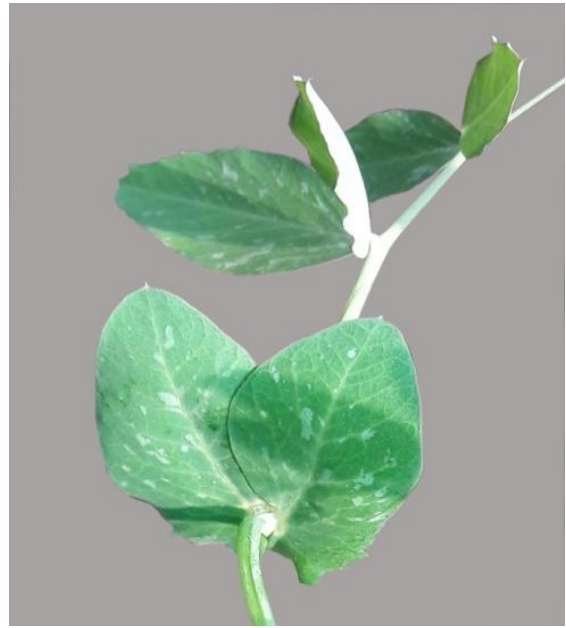

Fig.4 Stipule axil colour green

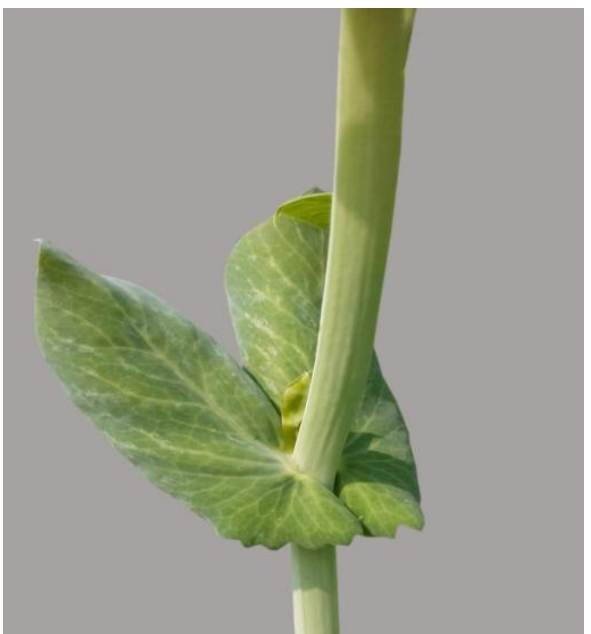

Table.1 Essential characters along with descriptor

\begin{tabular}{|c|c|c|c|c|c|c|}
\hline S. No & Characteristics & \multicolumn{5}{|c|}{ Strategies } \\
\hline 1. & Stem Anthocynin Coloration & Absent (1) & present (9) & & & \\
\hline 2. & Foliage Colour & Light Green (3) & Green (5) & $\begin{array}{c}\text { Dark Green } \\
\text { (7) }\end{array}$ & & \\
\hline 3. & Foliage Waxy Bloom & Absent (1) & present (9) & & & \\
\hline 4. & Leaflets & Absent (1) & present (9) & & & \\
\hline 5. & Leaf Axil Color & Green (1) & Purple (2) & & & \\
\hline 6. & Stipule: Rabit Eared & Absent (1) & present (9) & & & \\
\hline 7. & Stipule: Type & Normal (1) & Vestigial(3) & & & \\
\hline 8. & Flower Standard Petal colour & White (1) & Blue (2) & Pink (3) & $\operatorname{Red}(4)$ & Purple (5) \\
\hline 9. & Pod curvature & Absent (1) & Weak (3) & Medium (5) & Strong (7) & \\
\hline 10. & Pod shape of distal part & Pointed (1) & Blunt (9) & & & \\
\hline 11. & Seed shape & Spherical (1) & Cylindrical (2) & Dimpled (3) & & \\
\hline 12. & Seed surface & Smooth (1) & Wrinkled (2) & & & \\
\hline 13. & Seed cotyledon colour & Creamy(3) & Green (5) & Yellow (7) & & \\
\hline
\end{tabular}


Int.J.Curr.Microbiol.App.Sci (2018) 7(3): 2533-2542

Table.2 Characterization table of one hundred one lines as per note number given in DUS Guideline

\begin{tabular}{|c|c|c|c|c|c|c|c|c|c|c|c|c|c|c|}
\hline S. No. & Germplasm & 1 & 2 & 3 & 4 & 5 & 6 & 7 & 9 & 11 & 12 & 15 & 16 & 17 \\
\hline 1 & B-22 & 9 & 3 & 9 & 9 & 2 & 1 & 1 & 5 & 1 & 9 & 3 & 1 & 3 \\
\hline 2 & Shikha & 1 & 3 & 9 & 9 & 1 & 1 & 1 & 1 & 1 & 9 & 2 & 1 & 7 \\
\hline 3 & Rachna & 1 & 5 & 9 & 1 & 1 & 9 & 1 & 1 & 1 & 9 & 1 & 1 & 7 \\
\hline 4 & Jayanti & 1 & 5 & 9 & 9 & 1 & 1 & 1 & 1 & 1 & 9 & 1 & 1 & 7 \\
\hline 5 & VL-1 & 1 & 7 & 9 & 9 & 1 & 9 & 1 & 1 & 1 & 9 & 2 & 2 & 5 \\
\hline 6 & VL-3 & 1 & 3 & 9 & 9 & 1 & 9 & 1 & 1 & 3 & 9 & 2 & 2 & 3 \\
\hline 7 & P-3 & 1 & 5 & 9 & 1 & 1 & 1 & 1 & 1 & 1 & 9 & 1 & 1 & 7 \\
\hline 8 & RP-3 & 1 & 3 & 9 & 9 & 1 & 9 & 1 & 1 & 1 & 9 & 1 & 1 & 5 \\
\hline 9 & DDR-23 & 1 & 7 & 9 & 9 & 1 & 1 & 1 & 1 & 5 & 9 & 2 & 1 & 3 \\
\hline 10 & DDR-39 & 1 & 3 & 9 & 9 & 1 & 1 & 1 & 1 & 1 & 9 & 1 & 1 & 3 \\
\hline 11 & DDR-43 & 1 & 5 & 9 & 9 & 1 & 1 & 1 & 1 & 1 & 9 & 1 & 1 & 3 \\
\hline 12 & DDR-44 & 1 & 5 & 9 & 1 & 1 & 1 & 1 & 1 & 3 & 9 & 2 & 2 & 5 \\
\hline 13 & DDR-52 & 1 & 7 & 9 & 1 & 1 & 1 & 1 & 1 & 3 & 9 & 2 & 1 & 3 \\
\hline 14 & DDR-54 & 1 & 3 & 9 & 9 & 2 & 9 & 1 & 5 & 3 & 9 & 1 & 1 & 3 \\
\hline 15 & DDR-55 & 1 & 7 & 9 & 9 & 1 & 1 & 1 & 1 & 1 & 9 & 2 & 2 & 7 \\
\hline 16 & P 81-10 & 1 & 3 & 9 & 9 & 1 & 1 & 1 & 1 & 1 & 9 & 1 & 1 & 7 \\
\hline 17 & JP-885(Purple) & 1 & 7 & 9 & 9 & 2 & 1 & 1 & 5 & 1 & 9 & 3 & 2 & 5 \\
\hline 18 & Late Sown & 1 & 5 & 9 & 1 & 1 & 1 & 1 & 1 & 1 & 9 & 3 & 2 & 7 \\
\hline 19 & FP 9-539 & 1 & 5 & 9 & 1 & 1 & 1 & 1 & 1 & 3 & 9 & 1 & 1 & 3 \\
\hline 20 & FP 9-540 & 1 & 7 & 9 & 9 & 1 & 9 & 1 & 1 & 3 & 9 & 1 & 1 & 7 \\
\hline 21 & FP 7-562 & 1 & 5 & 9 & 1 & 1 & 1 & 1 & 1 & 5 & 9 & 1 & 1 & 3 \\
\hline 22 & FP 7-596 & 1 & 7 & 9 & 9 & 1 & 9 & 1 & 1 & 3 & 9 & 1 & 1 & 7 \\
\hline 23 & JM 91-01 & 1 & 3 & 9 & 9 & 1 & 1 & 1 & 1 & 1 & 9 & 1 & 1 & 7 \\
\hline 24 & KPMR-30 & 1 & 5 & 9 & 9 & 1 & 1 & 1 & 1 & 1 & 9 & 1 & 1 & 7 \\
\hline 25 & KPMR-302 & 1 & 3 & 9 & 9 & 1 & 1 & 1 & 1 & 1 & 9 & 1 & 1 & 7 \\
\hline 26 & KPMR-502 & 1 & 5 & 9 & 9 & 1 & 9 & 1 & 1 & 1 & 9 & 1 & 1 & 7 \\
\hline 27 & KPMR-503 & 1 & 5 & 9 & 9 & 1 & 9 & 1 & 1 & 1 & 9 & 1 & 1 & 7 \\
\hline 28 & KPMR-504 & 1 & 5 & 9 & 9 & 1 & 1 & 1 & 1 & 1 & 9 & 1 & 1 & 3 \\
\hline 29 & KPMR-400 & 1 & 5 & 9 & 9 & 1 & 1 & 1 & 1 & 1 & 9 & 2 & 2 & 5 \\
\hline 30 & KPMR-402 & 1 & 3 & 9 & 9 & 1 & 1 & 1 & 1 & 1 & 9 & 1 & 1 & 3 \\
\hline 31 & KPMR-420 & 1 & 3 & 9 & 9 & 1 & 1 & 1 & 1 & 1 & 9 & 1 & 1 & 7 \\
\hline 32 & KPMR-423 & 1 & 3 & 9 & 9 & 1 & 1 & 1 & 1 & 3 & 9 & 1 & 1 & 7 \\
\hline 33 & KPMR-427 & 1 & 5 & 9 & 1 & 1 & 1 & 1 & 1 & 1 & 9 & 1 & 1 & 7 \\
\hline 34 & KPMR-485 & 1 & 3 & 9 & 9 & 1 & 1 & 1 & 1 & 1 & 9 & 2 & 1 & 7 \\
\hline 35 & KPMR-486 & 1 & 3 & 9 & 9 & 1 & 1 & 1 & 1 & 1 & 9 & 1 & 1 & 3 \\
\hline 36 & KPMR-144 & 1 & 5 & 9 & 9 & 1 & 1 & 1 & 1 & 1 & 9 & 1 & 1 & 3 \\
\hline 37 & KPMR-327 & 1 & 5 & 9 & 9 & 1 & 1 & 1 & 1 & 3 & 9 & 1 & 1 & 3 \\
\hline 38 & KFP-151 & 1 & 5 & 9 & 9 & 1 & 1 & 1 & 1 & 1 & 9 & 1 & 1 & 3 \\
\hline 39 & DDP 99-14 & 1 & 5 & 9 & 1 & 1 & 1 & 1 & 1 & 3 & 9 & 1 & 1 & 3 \\
\hline 40 & HFP 94-12 & 1 & 5 & 9 & 1 & 1 & 1 & 1 & 1 & 1 & 9 & 2 & 2 & 7 \\
\hline 41 & HFP 94-13 & 1 & 5 & 9 & 1 & 1 & 1 & 1 & 1 & 3 & 9 & 1 & 1 & 7 \\
\hline 42 & HUVP-2 & 1 & 5 & 9 & 1 & 1 & 1 & 1 & 1 & 1 & 9 & 3 & 1 & 3 \\
\hline 43 & HUVP-12 & 1 & 3 & 9 & 9 & 1 & 1 & 1 & 1 & 1 & 9 & 1 & 1 & 7 \\
\hline 44 & HVP-2 & 1 & 5 & 9 & 1 & 1 & 9 & 1 & 1 & 3 & 9 & 2 & 1 & 7 \\
\hline 45 & NDVP-20 & 1 & 3 & 9 & 9 & 1 & 1 & 1 & 1 & 1 & 9 & 1 & 1 & 7 \\
\hline 46 & NDVP-4 & 1 & 3 & 9 & 9 & 1 & 1 & 1 & 1 & 1 & 9 & 1 & 1 & 7 \\
\hline 47 & LEP-227 & 1 & 3 & 9 & 9 & 1 & 1 & 1 & 1 & 1 & 9 & 1 & 1 & 7 \\
\hline 48 & LEP-260 & 1 & 5 & 9 & 9 & 1 & 1 & 1 & 1 & 1 & 9 & 1 & 1 & 3 \\
\hline 49 & JFP-27 & 1 & 5 & 9 & 9 & 1 & 1 & 1 & 1 & 1 & 9 & 1 & 1 & 7 \\
\hline 50 & JFP 99-25 & 1 & 3 & 9 & 9 & 1 & 1 & 1 & 1 & 1 & 9 & 1 & 1 & 3 \\
\hline 51 & PP-14 & 1 & 7 & 9 & 9 & 1 & 9 & 1 & 1 & 1 & 9 & 1 & 2 & 3 \\
\hline
\end{tabular}




\begin{tabular}{|c|c|c|c|c|c|c|c|c|c|c|c|c|c|c|}
\hline 52 & PP-86 & 1 & 7 & 9 & 9 & 1 & 9 & 1 & 1 & 3 & 9 & 1 & 1 & 3 \\
\hline 53 & PP-96 & 1 & 7 & 9 & 9 & 1 & 9 & 1 & 1 & 3 & 1 & 1 & 1 & 3 \\
\hline 54 & PP-155 & 1 & 7 & 9 & 9 & 1 & 9 & 1 & 1 & 1 & 9 & 1 & 2 & 3 \\
\hline 55 & HUP-2 & 1 & 7 & 9 & 1 & 1 & 1 & 1 & 1 & 1 & 9 & 1 & 1 & 3 \\
\hline 56 & HUDP-15 & 1 & 7 & 9 & 1 & 1 & 1 & 1 & 1 & 1 & 9 & 1 & 1 & 3 \\
\hline 57 & FP 14-67 & 1 & 7 & 9 & 9 & 2 & 9 & 1 & 5 & 1 & 9 & 3 & 2 & 3 \\
\hline 58 & FP 14-76 & 1 & 5 & 9 & 1 & 1 & 9 & 1 & 1 & 3 & 1 & 1 & 1 & 7 \\
\hline 59 & FP 14-82 & 1 & 5 & 9 & 9 & 1 & 9 & 1 & 1 & 3 & 9 & 2 & 2 & 7 \\
\hline 60 & Kalamatar & 1 & 5 & 9 & 9 & 2 & 1 & 1 & 5 & 3 & 1 & 3 & 1 & 5 \\
\hline 61 & FP 14-65 & 1 & 7 & 9 & 9 & 1 & 1 & 1 & 1 & 3 & 9 & 2 & 1 & 7 \\
\hline 62 & FP 14-67 & 9 & 7 & 9 & 9 & 2 & 9 & 1 & 5 & 3 & 9 & 3 & 2 & 3 \\
\hline 63 & FP 14-69 & 1 & 7 & 9 & 9 & 1 & 1 & 1 & 1 & 3 & 9 & 2 & 2 & 7 \\
\hline 64 & FP 14-71 & 1 & 7 & 9 & 9 & 1 & 9 & 1 & 1 & 1 & 9 & 2 & 1 & 7 \\
\hline 65 & FP 14-73 & 1 & 3 & 9 & 9 & 1 & 9 & 1 & 1 & 3 & 9 & 1 & 1 & 3 \\
\hline 66 & FP 14-75 & 1 & 7 & 9 & 9 & 1 & 9 & 1 & 1 & 1 & 9 & 2 & 1 & 3 \\
\hline 67 & FP 14-81 & 1 & 5 & 9 & 1 & 1 & 1 & 1 & 1 & 3 & 9 & 1 & 1 & 7 \\
\hline 68 & FP 14-82 & 1 & 7 & 9 & 9 & 1 & 1 & 1 & 1 & 3 & 9 & 2 & 2 & 3 \\
\hline 69 & FP $14-85$ & 1 & 3 & 9 & 9 & 1 & 1 & 1 & 1 & 1 & 9 & 1 & 1 & 3 \\
\hline 70 & FP 14-86 & 1 & 7 & 9 & 1 & 1 & 9 & 1 & 1 & 3 & 9 & 3 & 1 & 3 \\
\hline 71 & FP 14-87 & 1 & 7 & 9 & 1 & 1 & 1 & 1 & 1 & 1 & 9 & 1 & 1 & 3 \\
\hline 72 & FP 14-88 & 9 & 7 & 9 & 9 & 2 & 9 & 1 & 5 & 3 & 9 & 3 & 2 & 3 \\
\hline 73 & FP 14-89 & 1 & 7 & 9 & 9 & 1 & 1 & 1 & 1 & 3 & 9 & 3 & 2 & 3 \\
\hline 74 & FP 14-90 & 1 & 7 & 9 & 9 & 1 & 9 & 1 & 1 & 3 & 9 & 1 & 1 & 7 \\
\hline 75 & FP 14-36 & 1 & 7 & 9 & 9 & 1 & 9 & 1 & 1 & 3 & 9 & 2 & 2 & 3 \\
\hline 76 & FP 14-39 & 1 & 7 & 9 & 9 & 1 & 1 & 1 & 1 & 3 & 9 & 1 & 1 & 3 \\
\hline 77 & FP 14-41 & 1 & 7 & 9 & 9 & 1 & 1 & 1 & 1 & 1 & 9 & 2 & 2 & 7 \\
\hline 78 & FP 14-44 & 1 & 7 & 9 & 9 & 1 & 9 & 1 & 1 & 3 & 1 & 1 & 1 & 3 \\
\hline 79 & FP 14-46 & 1 & 7 & 9 & 9 & 1 & 9 & 1 & 1 & 1 & 9 & 3 & 2 & 3 \\
\hline 80 & FP $14-50$ & 1 & 5 & 9 & 9 & 1 & 1 & 1 & 1 & 3 & 9 & 1 & 1 & 7 \\
\hline 81 & FP 14-51 & 1 & 7 & 9 & 1 & 1 & 1 & 1 & 1 & 3 & 1 & 1 & 1 & 3 \\
\hline 82 & FP 14-54 & 1 & 7 & 9 & 9 & 1 & 1 & 1 & 1 & 1 & 9 & 3 & 2 & 7 \\
\hline 83 & FP 14-56 & 1 & 7 & 9 & 9 & 1 & 9 & 1 & 1 & 1 & 9 & 1 & 1 & 5 \\
\hline 84 & FP 14-15 & 1 & 7 & 9 & 9 & 1 & 9 & 1 & 1 & 1 & 9 & 1 & 1 & 3 \\
\hline 85 & FP 14-17 & 1 & 7 & 9 & 9 & 1 & 9 & 1 & 1 & 3 & 1 & 1 & 1 & 7 \\
\hline 86 & FP 14-21 & 1 & 3 & 9 & 1 & 1 & 9 & 1 & 1 & 1 & 9 & 1 & 1 & 7 \\
\hline 87 & FP $14-23$ & 1 & 7 & 9 & 9 & 1 & 9 & 1 & 1 & 1 & 1 & 1 & 1 & 5 \\
\hline 88 & FP 14-24 & 1 & 7 & 9 & 9 & 1 & 9 & 1 & 1 & 1 & 9 & 1 & 1 & 7 \\
\hline 89 & FP $14-25$ & 1 & 7 & 9 & 9 & 1 & 1 & 1 & 1 & 1 & 9 & 1 & 1 & 3 \\
\hline 90 & FP 14-26 & 1 & 7 & 9 & 9 & 1 & 1 & 1 & 1 & 1 & 9 & 1 & 1 & 7 \\
\hline 91 & FP 14-27 & 1 & 3 & 9 & 1 & 1 & 1 & 1 & 1 & 1 & 9 & 1 & 1 & 7 \\
\hline 92 & FP 14-28 & 1 & 3 & 9 & 9 & 1 & 9 & 1 & 1 & 1 & 9 & 1 & 1 & 7 \\
\hline 93 & FP 14-30 & 1 & 7 & 9 & 9 & 1 & 1 & 1 & 1 & 3 & 9 & 1 & 1 & 3 \\
\hline 94 & FP 14-32 & 1 & 7 & 9 & 9 & 1 & 1 & 1 & 1 & 3 & 9 & 1 & 1 & 7 \\
\hline 95 & FP 14-33 & 1 & 7 & 9 & 9 & 1 & 9 & 1 & 1 & 3 & 9 & 1 & 1 & 7 \\
\hline 96 & FP 14-34 & 1 & 7 & 9 & 9 & 1 & 9 & 1 & 1 & 1 & 9 & 1 & 1 & 7 \\
\hline 97 & IFP 99-25 & 1 & 7 & 9 & 9 & 1 & 1 & 1 & 1 & 1 & 9 & 1 & 1 & 7 \\
\hline 98 & FP 14-4 & 1 & 7 & 9 & 9 & 1 & 1 & 1 & 1 & 3 & 9 & 1 & 1 & 3 \\
\hline 99 & FP 14-5 & 1 & 7 & 9 & 9 & 1 & 9 & 1 & 1 & 3 & 9 & 1 & 1 & 3 \\
\hline 100 & FP 14-9 & 1 & 7 & 9 & 9 & 1 & 1 & 1 & 1 & 3 & 9 & 1 & 1 & 3 \\
\hline 101 & FP 14-13 & 1 & 7 & 9 & 9 & 1 & 1 & 1 & 1 & 3 & 9 & 1 & 1 & 7 \\
\hline
\end{tabular}

Note -1 to 9 alphabets are used here to tabulate the characteristics stratigies as per note number given in DUS guideline.

1-stem: anthocynin coloration, 2-foliage colour, 3-foliage waxy bloom, 4-leaf: leaflets, 5-leaf: axil color, 6-stipule: rabit eared, 7-stipule: type, 9-flower standard petal colour, 11-pod curvature, 12-pod shape of distal part, 15-seed shape, 16-seed surface, 17-seed: cotyledon colour 
Table.3 Grouping of pea germplasm in respect of pigmentation in plant parts

\begin{tabular}{|c|c|c|c|c|c|c|c|c|c|c|c|c|c|c|}
\hline \multirow[b]{2}{*}{ Group } & \multicolumn{2}{|c|}{ SAC } & \multicolumn{3}{|c|}{ FC } & \multicolumn{2}{|c|}{ FSPC } & \multicolumn{2}{|c|}{ LAC } & \multicolumn{3}{|c|}{$\mathrm{SCC}$} & \multirow{2}{*}{$\begin{array}{l}\text { Total } \\
\text { no. of } \\
\text { entries }\end{array}$} & \multirow[t]{2}{*}{ Name of lines } \\
\hline & $\begin{array}{l}\text { P- } \\
\text { SAC }\end{array}$ & $\begin{array}{l}\text { A- } \\
\text { SAC }\end{array}$ & $\begin{array}{l}\text { DG- } \\
\text { FC }\end{array}$ & $\begin{array}{l}\text { G- } \\
\text { FC }\end{array}$ & $\begin{array}{l}\text { LG- } \\
\text { FC }\end{array}$ & $\begin{array}{l}\text { P- } \\
\text { FSPC }\end{array}$ & $\begin{array}{l}\text { W- } \\
\text { FSPC }\end{array}$ & $\begin{array}{c}\text { G- } \\
\text { LAC }\end{array}$ & $\begin{array}{l}\text { P- } \\
\text { LAC }\end{array}$ & $\begin{array}{l}\text { C- } \\
\text { SCC }\end{array}$ & $\begin{array}{l}\text { G- } \\
\text { SCC }\end{array}$ & $\begin{array}{l}\text { Y- } \\
\text { SCC }\end{array}$ & & \\
\hline I & + & - & - & - & + & + & - & - & + & + & - & - & 1 & B-22 \\
\hline II & - & + & - & - & + & + & - & - & + & + & - & - & 1 & DDR 54 \\
\hline III & - & + & + & - & - & + & - & - & + & - & + & - & 1 & JP 885 (purple) \\
\hline IV & - & + & + & - & - & + & - & - & + & + & - & - & 1 & FP 14-67 \\
\hline $\mathbf{V}$ & - & + & - & + & - & + & - & - & + & - & + & - & 1 & Kalamatar \\
\hline VI & - & + & - & - & + & - & + & + & - & - & + & - & 1 & RP 3 \\
\hline VII & + & - & + & - & - & + & - & - & + & + & - & - & 2 & FP 14-67, FP 14-88 \\
\hline VIII & - & + & - & + & - & - & + & + & - & - & + & - & 2 & DDR 44, KPMR 400 \\
\hline IX & - & + & + & - & - & - & + & + & - & - & + & - & 3 & FP 14-56, FP 14-23, VL-1 \\
\hline $\mathbf{X}$ & - & + & - & - & + & - & + & + & - & + & - & - & 7 & $\begin{array}{l}\text { VL-3, DDR-39, KPMR-402, KPMR-486, JFP } \\
\text { 99-25, FP 14-73, FP 14-85 }\end{array}$ \\
\hline XI & - & + & - & + & - & - & + & + & - & + & - & - & 10 & $\begin{array}{l}\text { DDR-43, FP 9-539, FP 7-562, KPMR-504, } \\
\text { KPMR-144, KPMR-327, KFP-151, DDP 99-14, } \\
\text { HUVP-2, LEP-260 }\end{array}$ \\
\hline XII & - & + & - & - & + & - & + & + & - & - & - & + & 14 & $\begin{array}{l}\text { Shikha, P 81-10, JM 91-01, KPMR-302, KPMR- } \\
\text { 420, KPMR-423, KPMR-485, HUVP-12 , } \\
\text { NDVP-20, NDVP-4, LEP-227, FP 14-21, FP 14- } \\
\text { 27, FP 14-28 }\end{array}$ \\
\hline XIII & - & + & - & + & - & - & + & + & - & - & - & + & 16 & $\begin{array}{l}\text { Rachna, Jayanti, P-3, Late Sown, KPMR-30, } \\
\text { KPMR-427, KPMR-502, KPMR-503, HFP 94- } \\
\text { 12, HFP 94-13, HVP-2, JFP-27, FP 14-76, FP } \\
\text { 14-82, FP 14-81, FP 14-50 }\end{array}$ \\
\hline XIV & - & + & + & - & - & - & + & + & - & - & - & + & 17 & 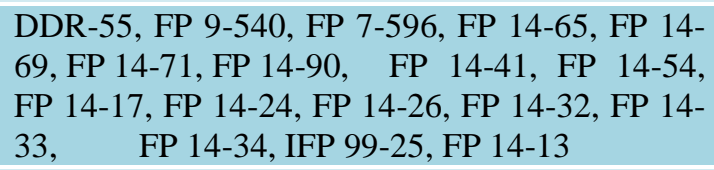 \\
\hline $\mathbf{X V}$ & - & + & + & - & - & - & + & + & - & + & - & - & 24 & $\begin{array}{l}\text { DDR-23, DDR-52, PP-14, PP-86, PP-96, PP- } \\
\text { 155, HUP-2, HUDP-15, FP 14-75, FP 14-82, FP } \\
\text { 14-86, FP 14-87, FP 14-89, FP 14-36, FP 14-39, } \\
\text { FP 14-44, FP 14-46, FP 14-51, FP 14-15, FP } \\
\text { 14-25, FP 14-30, FP 14-4, FP 14-5, FP 14-9 }\end{array}$ \\
\hline Total & & & & & & & & & & & & & 101 & \\
\hline
\end{tabular}

+ Pigmenmted, - Non-pigmented 
Plants usually present a dark green colour on high soil fertility and a light green one on low soil fertility (Fonseca et al., 2002), but those 7 germplasms having colour on leaf axil and flower standard petal both showing some different result in such condition. More study required on those germplasms for pointing out which are the traits that may be particularly referred to as indicators to determine an unstable expression of the phenotype of candidate variety (DUS guideline).

Present-Stem: Anthocynin Coloration ( $\mathrm{P}-$ SAC), Absent-Stem: Anthocynin Coloration (A-SAC), Dark Green-Foliage Colour (DGFC), Green-Foliage Colour (G-FC), Light Green-Foliage Colour (LG-FC), PurpleFlower Standard Petal colour (P-FSPC), White-Flower Standard Petal colour (WFSPC), Green-Leaf: Axil Colour (G-LAC), Purple-Leaf: Axil Colour (P-LAC), CreamySeed: cotyledon colour (C-SCC), Green-Seed: cotyledon colour (G-SCC), Yellow-Seed: cotyledon colour (Y-SCC).

Variation found higher for leaflets i.e., absent in 22 and present in 79 germplasm lines. The tendril-less trait is governed by a mutant allele named as tendril less ( $t l)$ which is semidominant (de Vilmorin, 1910; de Vilmorin and Bateson, 1911). It was not known if these were orthologous loci (Hofer et al., 2009). Tl is an wild type allele that carried by present day tendrilled peas as given by Lewis et al., 2005. Tendrils arose through acquisition of the $T l$ gene. According to Hofer et al., 2009 the pea tendril is developed by inhibiting complete laminar (leaflet) development which requires Unifoliata/LEAFY-mediated $T l$ expression (competitive dimerization with $T l$ ).

Seven germplasms having purple colour on leaf axil and flower standard petal both showing some different result in such condition. Chang (1979) suggested that purple pigmentation of rice plants had been lost during the process of evolution and genotypes with green types were evolved ones. So considering this reference may be this type of evolution had been in pea germplasms also.

Stipule growth and development is directed by interaction of coch and st genes in Pisum sativum. But the coch and st genes functions are inter-related in stipule development are not closely clustered but widely dispersed in the genome (Mishra et al., 2009). Stipule remains small blade type of structure due to natural mutant called st (stipule reduced) (Pellew et al., 1923). This cannot normally be seen as $T l$ does not act on the stipules, but the interaction of coch and tendril-less $(t l)$ genes in the compound stipules reveals that the expression of the wild-type $T l$ gene is reduced at the base nodes of the plant so stipule remain functionless or vestigial, supporting the theories of gradients of gene action. (Yaxley et al., 2001).

Flower Standard Petal colour and seed coat colour concept is elaborated by various researchers earlier. Genes corresponding to either MYB or basic-helix-loop-helix (bHLH) transcription factors or WD40 proteins are known regulators of anthocyanin biosynthesis (Koes et al., 2005) and previous investigations in pea have suggested that the white flower character determined by the recessive allele $a$, is due to the disruption of a regulatory gene (Harker et al., 1990). They found that the A gene encodes a transcription factor (bHLH) - a protein - which controls anthocyanin synthesis and hence produces purple flowers and coloured seed coats (Hellens et al., 2010). They also identified a mutation (a) which produces white flowered pea lines. This mutant fails to manufacture the transcription factor and hence anthocyanin production is interrupted so the flowers appear white and the seed coats are not pigmented. The A2 gene encodes a WD40 protein that is part of an evolutionarily 
conserved regulatory complex (Hellens et al., 2010). We found that another gene which controls flower colour - $B$ - encodes an enzyme (defective flavonoid 3', 5'hydroxylase) and confers pink flower color biosynthesis (Moreau et al., 2012). Recessive $b$ mutants cannot produce this enzyme; hence the flowers lack two major anthocyanins and exhibit a pink appearance instead of purple.

Rayner et al., 2017 work described here identifies a novel source of genetic variation controlling wrinkled-seeded phenotypes in pea germplasm. The $\mathrm{r}$ locus or a mutation at a second genetic locus, rb, affecting the gene encoding the large subunit of Adenosine diphosphoglucose (ADP-glucose) pyro phosphorylase. The novel wrinkled-seeded phenotype identified in JI 2110 is maternally determined and is likely to be controlled by two genetic loci. Metabolite profiling revealed changes in myoinositol concentration in JI 2110, particularly in the testa, suggesting an explanation for the seed phenotype.

The inflated versus constricted pod phenotype refers to the presence or absence of a layer of lignified cells (sclerenchyma) adjoining the epidermis of the pod wall and is referred to as parchment (Ellis et al., 2011). Absence of this cell layer leads to change in pod curvature at maturity.

Further, the identified variability and unique features can be selected for developing varieties with unique identification, diverse traits and broaden genetic base. Above study revealed some of distinct characteristics of varieties and indicated that morphological variations exist in collected varieties due to variation in genetic makeup and but more study required on other traits also which are not studied at genetic level. The unique pure lines possessed exclusive variability and unique features which need safe conservation and sustainable utilization in future pea varieties development for issues like intellectual property rights.

\section{References}

Bajracharya, J., Steele, K. A., Jarvis, D. I., Sthapit, B. R. and Witcombe, J. R. (2006). Rice landrace diversity in Nepal: variability of agro-morphological traits and SSR markers in landraces from a high-altitude site. Fields Crop Research 95: 327-335.

Baranger, A., Aubert, G., Arnau, G., Laine, A. L., Denoit, G., Potier, J., Weinachter, C., Lejeune-Henaut, I., Lallemand, J. and Burstin, J. (2004). Genetic diversity within Pisum sativum using protein- and PCRbased markers. Theor. Appl. Genet., (108): 1309-1321.

Basaiwala, P., Rastogi, N. K. and Parikh, M. (2013). Genetic variability and character association in field pea (Pisum sativum L.) genotypes. The Asian journal of horticulture volume 8 (1): 288-291.

Chang, T. T. (1979). Rice, O. sativa and O. glaberrima (Graminae-Oryzeae) Evolution of crop plants. Longmans Ltd. W-7, Edi. N.W. Simonds, Pp98-104.

De Vilmorin, P. \& Bateson, W. (1911). A case of gametic coupling in Pisum, Proc R Soc London Series B Biol Sci, (84) 9-11.

De Vilmorin, P. (1910). Experiments on Mendelian inheritance (transl. from French). C. R. Acad. Sci. Paris 2: 548-551.

Ellis, T. H. N. (2011). Pisum. In: Kole C. (ed.): Wild Crop Relatives: Genomic and Breeding Resources (Legume Crops and Forages). Springer Verlag, BerlinHeidelberg, 237-248.

Fonseca, J. R., Cutrim, V. A. and Rangel, P. H. N. 2002. Morpho-Agronomic Descriptors and Phenology of the Lowland Rice Cultivars. Embrapa, Brasília, DF, Brazil (in Portuguese).

Gopalakrishnan, T. R. (2007). Vegetable crops. New India Publishing Agency (India).

Harker, C. L., Ellis, T. H. N. and Coen. E. S. (1990). Identification and Genetic- 
Regulation of the Chalcone Synthase Multigene Family in Pea. Plant Cell 2: 185-194.

Hellens, R. P., Moreau, C., Lin-Wang, K., Schwinn, K. E., Thomson, S. J., Fiers, M. W. E. J., Frew, T. J., Murray, S. R., Hofer, J. M. I. and Jacobs, J. M. E. (2010). Identification of Mendel's white flower character. PLoS ONE 5: e13230.

Hofer, J., Turner, L., Moreau, C., Ambrose, M., Isaac, P., Butcher, S., Weller, J., Dupin, A., Dalmais, M. and Signor, C.L. (2009). Bendahmane A. and Ellis N. Tendril-less Regulates Tendril Formation in Pea Leaves. The Plant Cell, Vol. 21: 420-428.

Koes, R., Verweij, W. and Quattrocchio, F. (2005). Flavonoids: a colorful model for the regulation and evolution of biochemical pathways. Trends in Plant Science 10:236242.

Lewis, G. P., Schrire, B., Mackinder, B., and Lock, M. (2005). Legumes of the World. (Richmond, UK: Kew Publishing).

Mishra, R. K., Kumar, A., Chaudhary, S. and Kumar, S. (2009). Mapping of the multifoliate pinna (mfp) leaf-blade morphology mutation in grain pea Pisum sativum. J. Genet. 88: 227-232.

Moreau, C., Ambrose, M. J., Turner, L., Hill, L., Ellis, T. H. N. and Hofer, J. M. I. (2012). The $b$ Gene of Pea Encodes a Defective Flavonoid 39, 59-Hydroxylase, and Confers Pink Flower Color. Plant Physiology, Vol. 159, pp. 759-768.

Nisar, M., Ghafoor, A. and Khan, M. R. (2011). Phenotypic variation in the agronomic and morphological traits of Pisum sativum L. germplasm obtained from different parts of the world. Genetika 47: 25-31.

Parihar, A. K., Dixit, G. P., Pathak, V. and Singh, D. (2014). Assessment of the genetic components and trait associations in diverse set of field pea (Pisum sativum L.) genotypes. Bangladesh Journal of Botany 43 (3): 323-330.

Pellew, C. and Sverdrup, A. (1923). New observations on the genetics of peas (Pisum sativum).J. Genet.13, 125-131.

Raut, V. M. (2003). Qualitative genetics of Soyabean-a review. Soybean Research, 1: $1-28$.

Rayner, T., Moreau, C., Ambrose, M., Isaac, P.G., Ellis, N. and Domoney, C. (2017). Genetic Variation Controlling Wrinkled Seed Phenotypes in Pisum: How Lucky Was Mendel? Int. J. Mol. Sci., 18, 1205.

Tar'an, B., Zhang, C., Warkentin, T. D., Tullu, A., Vandenberg, A. (2005). Genetic diversity among varieties and wild species accessions of pea (Pisum sativum L.) based on molecular markers, and morphological and physiological characters. Genome, 48, 257-272.

UPOV, (1990): Guidelines for the Conduct of Tests for Distinctness, Homogeneity, and Stability. Document UPOV TG/4/7. Geneva, Switzerland

Yaxley, J. L., Jablonski, W. \& Reid, J. B. (2001). Leaf and flower development in pea (Pisum sativum L.): Mutants cochleata and unifoliata, Ann Bot, (88) 225.

\section{How to cite this article:}

Lokesh Gour, R.K. Dubey, S.K. Singh and Sharad Tiwari. 2018. Morpho-Diversification Study of Indigenous Gene-Pool of Pisum sativum L. at Madhya Pradesh, India. Int.J.Curr.Microbiol.App.Sci. 7(03): 2533-2542. doi: https://doi.org/10.20546/ijcmas.2018.703.293 